\title{
Observation of Properties of Primary and Secondary Cosmic Rays by the Alpha Magnetic Spectrometer on the International Space Station
}

\author{
Alberto Oliva ${ }^{1, *}$ on behalf of the AMS-02 Collaboration \\ ${ }^{1}$ Centro de Investigaciones Energéticas, Medioambientales y Tecnológicas (CIEMAT), E-28040 Madrid, Spain
}

\begin{abstract}
The Alpha Magnetic Spectrometer (AMS-02) is a wide acceptance high-energy physics experiment installed on the International Space Station in May 2011 and operating continuously since then. With a collection rate of approximately $1.7 \times 10^{10}$ events/year, and the combined identification capabilities of 5 independent detectors, AMS-02 is able to precisely separate cosmic rays light nuclei $(1 \leq Z \leq 8)$. Knowledge of the precise rigidity dependence of the light nuclei fluxes is important in understanding the origin, acceleration, and propagation of cosmic rays. AMS-02 collaboration has recently released the precise measurements of the fluxes of light nuclei as a function of rigidity (momentum/charge) in the range between $2 \mathrm{GV}$ and $3 \mathrm{TV}$. Based on the observed spectral behaviour, the light nuclei can be separated in three distinct families: primaries (hydrogen, helium, carbon, and oxygen), secondaries (lithium, beryllium, and boron), and mixed (nitrogen). Spectral indices of all light nuclei fluxes progressively harden above $100 \mathrm{GV}$. Primary cosmic ray fluxes have an identical hardening above $60 \mathrm{GV}$, of about $\gamma=0.12 \pm 0.04$. While helium, carbon and oxygen have identical spectral index magnitude, the hydrogen spectral index shows a different magnitude, i.e. the primary-to-primary $\mathrm{H} / \mathrm{He}$ ratio is well described by a single power law above $45 \mathrm{GV}$ with index $-0.077 \pm 0.007$. Secondary cosmic ray fluxes have identical rigidity dependence above $30 \mathrm{GV}$. Secondary cosmic rays all harden more than primary species, and together all secondary-to-primary ratios show a hardening difference of $0.13 \pm 0.03$. Remarkably, the nitrogen flux is well described over the entire rigidity range by the sum of the primary flux equal to $9 \%$ of the oxygen flux and the secondary flux equal to $62 \%$ of the boron flux.
\end{abstract}

A precise knowledge of cosmic ray nuclei with charge $1 \leq Z \leq 8$ spectra in the GV-TV region provides important insights to the origin, acceleration, and subsequent propagation processes of cosmic rays in the Galaxy. Hydrogen, helium, carbon, and oxygen are primary cosmic rays and are thought to be mainly produced and accelerated in astrophysical sources. Lithium, beryllium, and boron nuclei are secondary cosmic rays and are thought to be produced by the collisions of cosmic rays with the interstellar medium. Nitrogen is thought to have significant primary and secondary components [1].

Over the last 30 years there have been many measurements of light nuclei fluxes [2-17]. Typically the measurements of boron, carbon and oxygen fluxes fluxes have errors larger than $15 \%$ at $100 \mathrm{GV}$, while lithium and beryllium fluxes have errors larger than $50 \%$ at $100 \mathrm{GV}$. These measurements showed the possible existence of a hardening in the primary cosmic rays [3-5]. The AMS-02 Collaboration has reported measurement of light nuclei spectra with atomic number $1 \leq Z \leq 8$ in the rigidity range from $2 \mathrm{GV}$ to $3 \mathrm{TV}$, based on 2.5 and 5 years of operations in Ref. [18-23], showing the existence of a progressive hardening above $100 \mathrm{GV}$ on all light nuclei species. These precision measurements, with a typical total error of 1$4 \%$ at $100 \mathrm{GV}$, allow the investigation of the nature of the hardening. For example, if the hardening in cosmic rays is

\footnotetext{
*e-mail: alberto.oliva@cern.ch
}

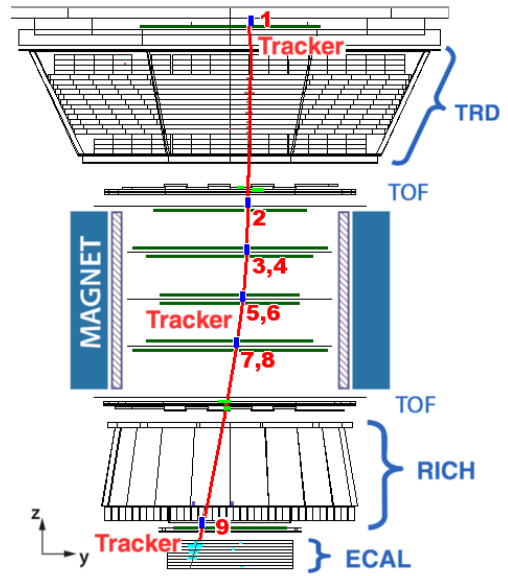

Figure 1. The layout of the AMS-02 detector. It consists of nine planes of precision silicon tracker, a transition radiation detector (TRD), four planes of time of flight counters (TOF), a permanent magnet, an array of anticoincidence counters (ACC), surrounding the inner tracker, a ring imaging Čerenkov detector (RICH), and an electromagnetic calorimeter (ECAL). A low energy event traversing the AMS-02 detector is superimposed.

related to the injected spectra at their source, then similar hardening is expected for both secondary and primary cosmic rays [24]. However, if the hardening is related to prop- 


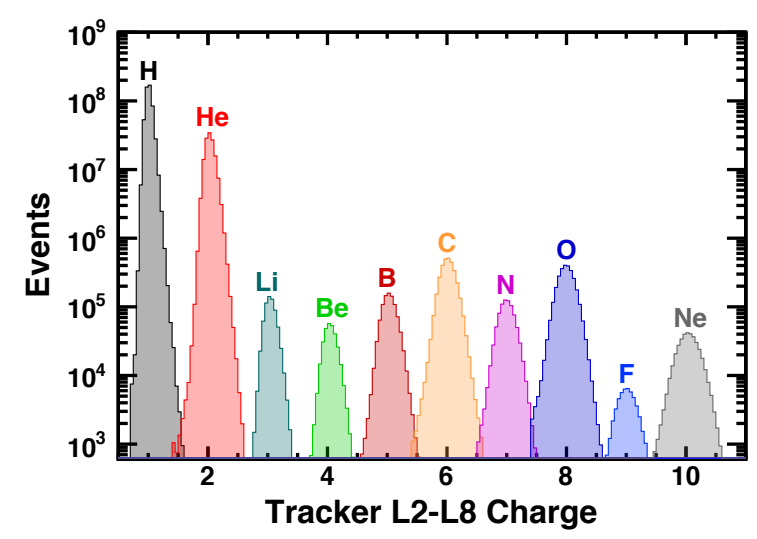

Figure 2. Distribution of the charge measured with the inner tracker L2-L8 for samples from $\mathrm{Z}=1$ to $\mathrm{Z}=10$ selected by a clean selection of charge measured with L1, the upper TOF, and the lower TOF in the rigidity range from $4 \mathrm{GV}$ to $38.9 \mathrm{GV}$. As seen, chemical species are well separated by the use of the inner tracker alone.

agation properties in the Galaxy then, a stronger hardening is expected for the secondary with respect to the primary cosmic rays [25].

The AMS-02 detector is a general purpose high-energy particle physics detector. It was installed on the International Space Station on 19 May 2011 to conduct a unique long duration mission ( $>20$ years) of fundamental physics research in space. The layout and description of the AMS-02 detector are presented in Ref. [26] and shown in Fig. 1. The key elements used in this measurement are the permanent magnet [27], the silicon tracker [28], and the four planes of time of flight (TOF) scintillation counters [29]. AMS-02 also contains a transition radiation detector (TRD), a ring imaging Čerenkov detector (RICH), an electromagnetic calorimeter (ECAL), and an array of 16 anticoincidence counters.

The tracker is composed of 9 layers of silicon doubleside micro-strip sensors. There is one layer on top of AMS (L1), 7 layers inside the magnet (L2 to L8) constituting the inner tracker, and one layer on the bottom of AMS on top of the calorimeter (L9). The tracker accurately determines the trajectory and absolute charge $|Z|$ of cosmic rays by multiple measurements of the coordinates and energy loss. Together, the tracker and the magnet measure the rigidity $R$ of charged cosmic rays. With a magnetic field of 1.4 $\mathrm{kG}$, the maximum detectable rigidity, i.e. the rigidity for which $\Delta R / R=100 \%$, is $2(3) \mathrm{TV}$ for $Z=1(2 \leq Z \leq 8)$ over the $3 \mathrm{~m}$ lever arm from L1 to L9. Each layer of the tracker also provides an independent measurement of the absolute value of the charge $|Z|$ of the cosmic ray. The charge resolution of the layers of the inner tracker together is $\Delta Z / Z<5 \%$ for $1 \leq Z \leq 8$. The charge separation achieved with the inner tracker charge is shown in Fig. 2.

Two planes of TOF counters are located above the magnet, and two planes are located below it. The difference in time between the upper TOF and the lower TOF, together with the track length measurement from tracker,

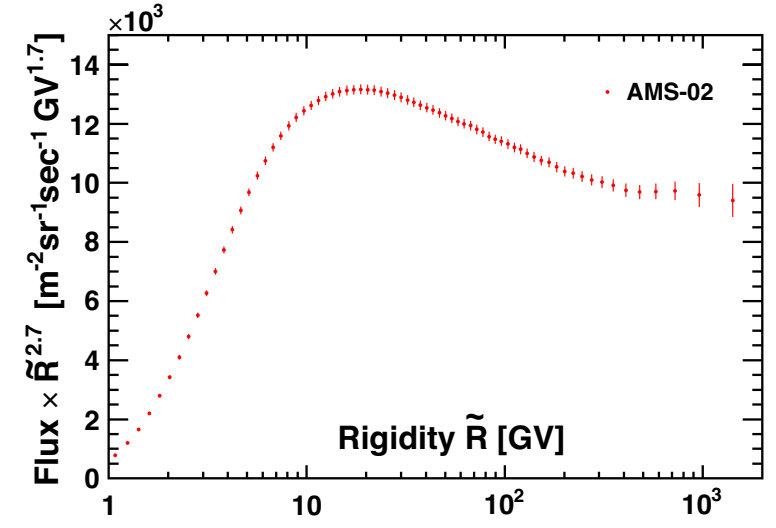

Figure 3. The AMS hydrogen flux multiplied by $\tilde{R}^{2.7}$ as a function of rigidity [18].

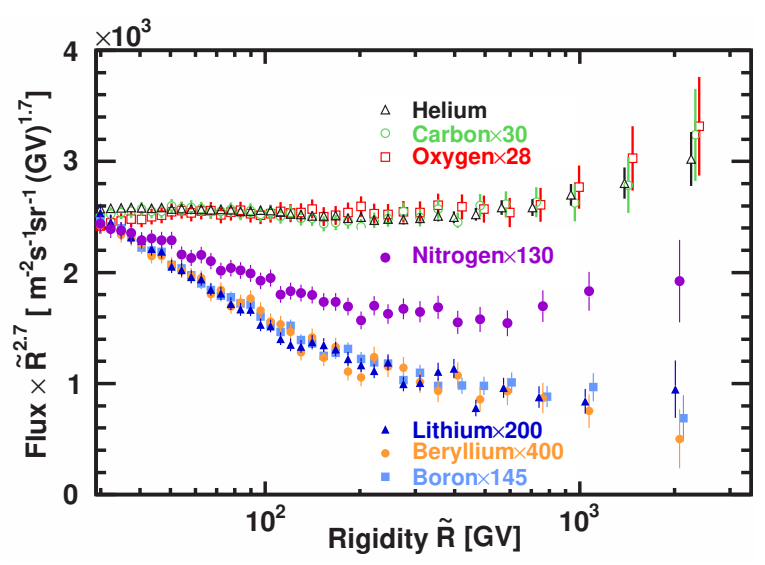

Figure 4. The AMS light nuclei $(2 \leq Z \leq 8)$ multiplied by $\tilde{R}^{2.7}$ as functions of rigidity above $30 \mathrm{GV}$ [21-23]. For display purposes only, the carbon, oxygen, lithium, beryllium, boron, and nitrogen fluxes were rescaled as indicated. For clarity, the horizontal positions of the helium, oxygen, lithium, and boron data points above $400 \mathrm{GV}$ are displaced. As seen, the three secondary fluxes have identical rigidity dependence above $30 \mathrm{GV}$ as do the three primary fluxes above $60 \mathrm{GV}$, but they are different from each other. The rigidity dependence of the nitrogen flux is distinctly different from the dependence of both the primary fluxes and the dependence of the secondary fluxes.

measure the particle velocity $(\beta=v / c)$. The velocity resolution has been measured to be $\Delta \beta / \beta=0.04(0.02) \times \beta$ for $Z=1(2 \leq Z \leq 8)$ particles. The time difference discriminates between upward- and downward-going particles. The pulse heights of the two upper layers, are combined to provide an independent measurement of the charge. The pulse heights from the two lower planes are combined to provide another independent charge measurement with a similar accuracy. Since launch in May 2011, all the detectors have been monitored constantly and calibrated periodically to ensure steady performances.

In the first 30 months AMS collected $8.5 \times 10^{10}$ cosmic ray events. The effective data collection time includes only those seconds during which the detector was in normal operating conditions. Events are required to be downward going, to have a reconstructed track in the inner tracker, 
and to pass through L1. In the highest rigidity region, $R>1 \mathrm{TV}$ the track is also required to pass through $\mathrm{L} 9$. Track fitting quality criteria such as a $\chi^{2} /$ d.o.f. $<10$ in the bending coordinate are applied. The measured rigidity is required to be greater than a factor of 1.2 times the maximum geomagnetic cutoff within the AMS field of view. The cutoff was calculated by backtracing particles from the top of AMS out to 50 Earth's radii [30] using the most recent IGRF [31] geomagnetic model. Charge measurements on tracker L1, the inner tracker (shown in Fig. 2), the upper TOF, and, for $R>1 \mathrm{TV}$, tracker $\mathrm{L} 9$ are required to be compatible with charge $Z$ from 1 to 8 . Complete descriptions of the event selection and background subtraction for each nucleus are reported in Ref. [18-23].

The isotropic flux $\Phi_{i}^{Z}$ for the species with charge $Z$ in the i-th rigidity bin $R_{i}, R_{i}+\Delta R_{i}$ is:

$$
\Phi_{i}^{Z}=\frac{N_{i}^{Z}}{A_{i}^{Z} \epsilon_{i}^{Z} T_{i} \Delta R_{i}}
$$

where $N_{i}^{Z}$ is the number of events after background subtraction and corrected for bin-to-bin rigidity migration, by the use of an unfolding procedure that uses the rigidity resolution function obtained from simulation; $A_{i}^{Z}$ is the effective acceptance calculate with the simulation and corrected for the small differences between data and simulation for reconstruction and selection efficiencies; $\epsilon_{i}^{Z}$ is the trigger efficiency measured from data with the unbiased trigger events (is $>90 \%$ for hydrogen, $>95 \%$ for helium and $>98 \%$ for $\mathrm{Z}>2$ ); $T_{i}$ is the collection time. Details about the unfolding procedure and of the acceptance calculation are described in Ref. [18-23].

Much effort has been spent evaluating the total systematic errors. These errors can be separated into several contributions: from the trigger efficiency and acceptance estimation; background contamination; the rigidity resolution function and unfolding; the absolute rigidity scale. Total error does not exceed $4 \%$ for any flux at $100 \mathrm{GV}$. The flux has been validated using different detector geometries and time periods. Several independent analyses were performed on the same data sample by different study groups and the results of those analyses are consistent to each other. The error estimation and the flux verification is reported in Ref. [18-23].

Figure 3 shows the hydrogen flux as function of rigidity with the total errors, the sum in quadrature of statistical and systematic errors. In this and the subsequent figures, the points are placed along the abscissa at $\tilde{R}$ calculated for a flux $\propto R^{-2.7}$. In figure 4 fluxes of cosmic ray nuclei with $2 \leq Z \leq 8$ are shown. All fluxes progressively harden above $100 \mathrm{GV}$. The three secondary fluxes have identical rigidity dependence above $30 \mathrm{GV}$ as do the three primary fluxes above $60 \mathrm{GV}$, but they are different from each other. The rigidity dependence of the nitrogen flux is distinctly different from the dependence of both the primary fluxes and the dependence of the secondary fluxes.

To obtain the detailed variation of the spectral index $\gamma^{Z}$ with rigidity in a model independent way, $\gamma^{Z}$ is calculated from

$$
\gamma^{Z}=\mathrm{d}\left[\log \left(\Phi^{Z}\right)\right] / \mathrm{d}[\log (R)]
$$

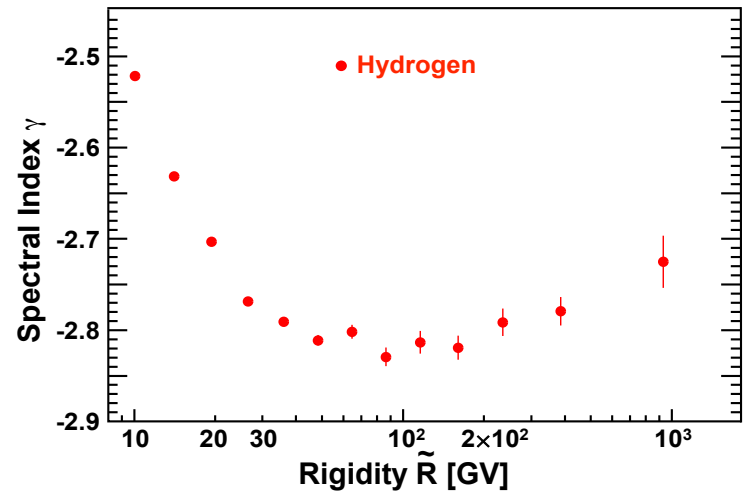

Figure 5. The dependence of the AMS hydrogen flux spectral index $\gamma$ on rigidity $\tilde{R}[18]$.

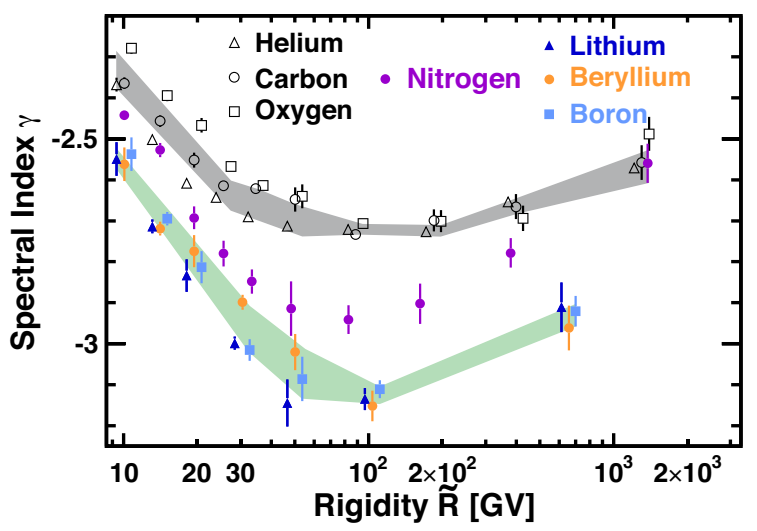

Figure 6. The dependence of the AMS light nuclei $(2 \leq Z \leq 8)$ spectral indices $\gamma$ on rigidity $\tilde{R}$ [21-23]. For clarity, the horizontal positions of the lithium and boron data points, and helium and oxygen data points are displaced with respect to the beryllium and carbon data points, respectively. The shaded regions are to guide the eye. The magnitude and the rigidity dependence of the secondaries lithium, beryllium, and boron spectral indices are nearly identical, but distinctly different from the rigidity dependence of the primaries helium, carbon, and oxygen spectral indices. Above $200 \mathrm{GV}$ the secondary fluxes all harden more than the primary fluxes. The nitrogen spectral index is situated between the primary and secondary cosmic ray spectral indices, hardens rapidly with rigidity above $\sim 100 \mathrm{GV}$ and becomes identical to the spectral indices of the primary cosmic rays above $\sim 700 \mathrm{GV}$.

over independent rigidity intervals with a variable width to have sufficient sensitivity to determine $\gamma^{Z}$. As seen in Fig. 5 and Fig. 6, all spectral indices vary with rigidity and progressively harden with rigidity above $100 \mathrm{GV}$. The spectral indices of helium, carbon and oxygen are nearly identical above $60 \mathrm{GV}$. Hydrogen shows a similar hardening to the $\mathrm{He}, \mathrm{C}$ and $\mathrm{O}$ above $45 \mathrm{GV}$ one, but the magnitude of the spectral index is different. Lithium, beryllium and boron spectra are also identical above $30 \mathrm{GV}$. Above $200 \mathrm{GV}, \mathrm{Li}, \mathrm{Be}$, and B harden more than $\mathrm{H}, \mathrm{He}, \mathrm{C}$, and $\mathrm{O}$. Nitrogen shows a distinctly different behaviour.

To examine the difference between the rigidity dependence of the primary species, in particular among hydro- 


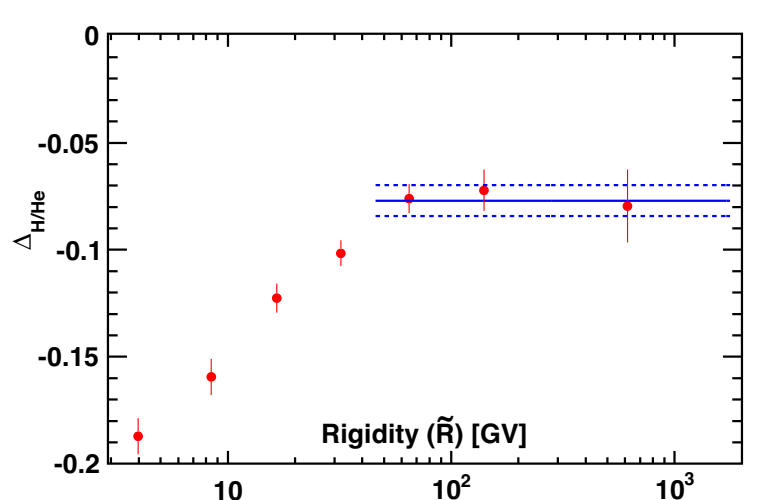

Figure 7. The rigidity dependence of the $\mathrm{H} / \mathrm{He}$ spectral index $\Delta_{\mathrm{H} / \mathrm{He}}$ as measured by AMS [19]. As seen, $\Delta_{\mathrm{H} / \mathrm{He}}$ increases up to about $45 \mathrm{GV}$. Above $45 \mathrm{GV}$ it becomes constant at $\Delta_{\mathrm{H} / \mathrm{He}}=$ $-0.077 \pm 0.007$ as indicated by the solid blue line.

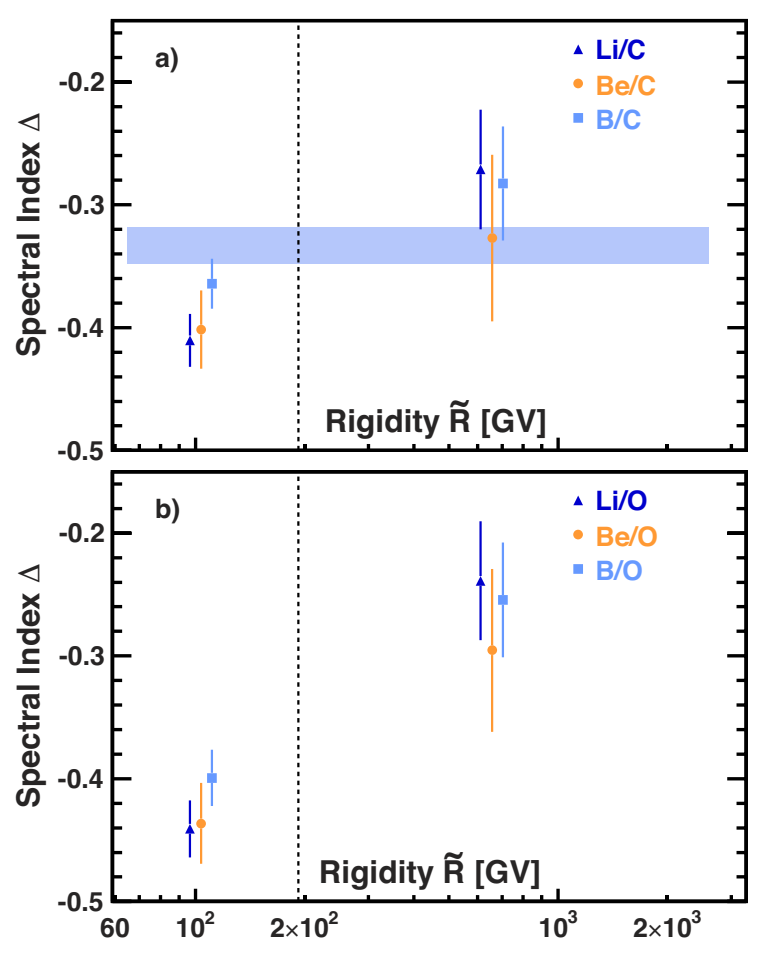

Figure 8. The AMS secondary to primary flux ratio spectral indices as functions of rigidity for $(\mathrm{a}) \mathrm{Li} / \mathrm{C}, \mathrm{Be} / \mathrm{C}$, and $\mathrm{B} / \mathrm{C}$ and for (b) $\mathrm{Li} / \mathrm{O}, \mathrm{Be} / \mathrm{O}$, and $\mathrm{B} / \mathrm{O}$. The horizontal band indicates the fit to the $\mathrm{B} / \mathrm{C}$ ratio from our previous publication [20]. On average, the spectral indices of $\mathrm{Li} / \mathrm{C}, \mathrm{Be} / \mathrm{C}, \mathrm{B} / \mathrm{C}, \mathrm{Li} / \mathrm{O}, \mathrm{Be} / \mathrm{O}$, and $\mathrm{B} / \mathrm{O}$ above $200 \mathrm{GV}$ exhibit a hardening of $0.13 \pm 0.03$.

gen and helium, the primary-to-primary $\mathrm{H} / \mathrm{He}$ ratio was computed [19]. The contribution of individual sources to the systematic error are added in quadrature to arrive at the total systematic uncertainty. The correlations in the systematic errors from the unfolding and the absolute rigidity scale between the hydrogen and helium fluxes have been accounted for in calculating the corresponding systematic errors of the $\mathrm{H} / \mathrm{He}$ ratio. Figure 7 shows the detailed variation the $\mathrm{H} / \mathrm{He}$ spectral index $\Delta_{\mathrm{H} / \mathrm{He}}=$ $\mathrm{d} \log (\mathrm{H} / \mathrm{He}) / \mathrm{d} \log (R)$ over non-overlapping rigidity inter-

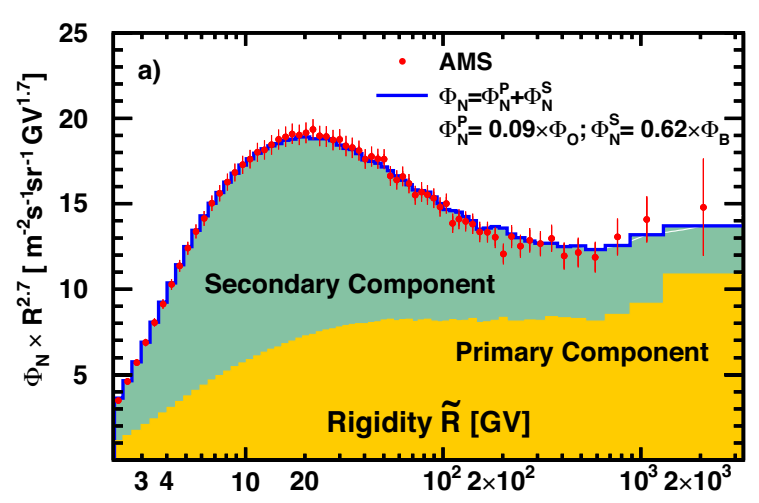

Figure 9. The AMS nitrogen flux $\Phi_{\mathrm{N}}$ fit to the weighted sum of the oxygen flux $\Phi_{\mathrm{O}}$ and the boron flux $\Phi_{\mathrm{B}}$ over the entire rigidity range [23].

vals. Above $45 \mathrm{GV}$ the $\mathrm{H} / \mathrm{He}$ spectral index becomes constant at $\Delta_{\mathrm{H} / \mathrm{He}}=-0.077 \pm 0.007$, i.e. hydrogen and helium have identical hardening above $200 \mathrm{GV}$, and the $\mathrm{H} / \mathrm{He}$ ratio measured by AMS is well fit with a single power law of index -0.077 above $45 \mathrm{GV}$.

To examine the difference between the rigidity dependence of primary and secondary cosmic rays in detail, the secondary-to-primary ratios of the lithium, beryllium, and boron fluxes to the carbon and oxygen fluxes were computed. The detailed variations with rigidity of the spectral indices $\Delta$ of each flux ratio were obtained in a model independent way using $\Delta=\mathrm{d}\left[\log \left(\Phi_{\mathrm{S}} / \Phi_{\mathrm{P}}\right)\right] / \mathrm{d}[\log (R)]$ where $\Phi_{\mathrm{S}} / \Phi_{\mathrm{P}}$ are the ratios of the secondary to primary fluxes over rigidity intervals [60.3-192] GV and [192-3300] GV and shown in Fig 8. Above $200 \mathrm{GV}$ these spectral indices exhibit an average hardening of $0.13 \pm 0.03$. This verifies that at high rigidities the secondary cosmic rays harden more than the primary cosmic rays. The additional hardening of secondary cosmic rays is consistent with expectations when the hardening of cosmic ray fluxes is due to the propagation properties in the Galaxy [25].

To obtain the fractions of the primary $\Phi_{\mathrm{N}}^{\mathrm{P}}$ and secondary $\Phi_{\mathrm{N}}^{\mathrm{S}}$ components in the nitrogen flux $\Phi_{\mathrm{N}}=\Phi_{\mathrm{N}}^{\mathrm{P}}+\Phi_{\mathrm{N}}^{\mathrm{S}}$, a fit of $\Phi_{N}$ to the weighted sum of a characteristic primary cosmic ray flux, namely oxygen $\Phi_{\mathrm{O}}[21]$, and of a characteristic secondary cosmic ray flux, namely boron $\Phi_{B}$ [22], was performed over the entire rigidity range, as shown in Fig. 9. The fit yields $\Phi_{\mathrm{N}}^{\mathrm{P}}=(0.090 \pm 0.002) \times \Phi_{\mathrm{O}}$ and $\Phi_{\mathrm{N}}^{\mathrm{S}}=(0.62 \pm 0.02) \times \Phi_{\mathrm{B}}$. The contribution of the secondary component in the nitrogen flux drops from $70 \%$ at a few GV to below $30 \%$ above $1 \mathrm{TV}$. The observation that the nitrogen flux can be fitted over a wide rigidity range as the simple linear combination of primary and secondary fluxes permits the determination of the N/O abundance ratio at the source without the need to consider the Galactic propagation of cosmic rays.

To compare AMS results with previous measurements a procedure to convert the fluxes from rigidity to the kinetic energy per nucleon $E_{K}$ is used, see Ref. [18-23]. The atomic mass numbers, averaged by isotopic composition obtained from AMS low energy measurements are used for the conversion. The systematic errors on the fluxes 


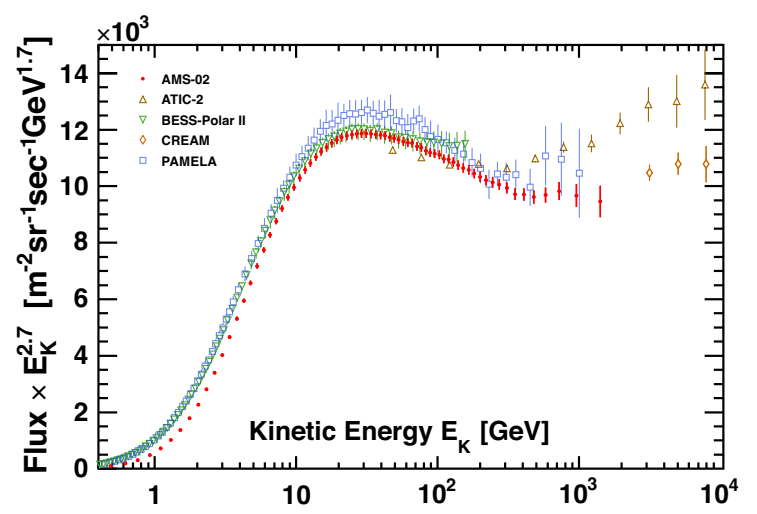

Figure 10. The AMS hydrogen flux [18] multiplied as function of kinetic energy per nucleon $E_{K}$ multiplied by $E_{K}^{2.7}$ together with earlier measurements [2-5].

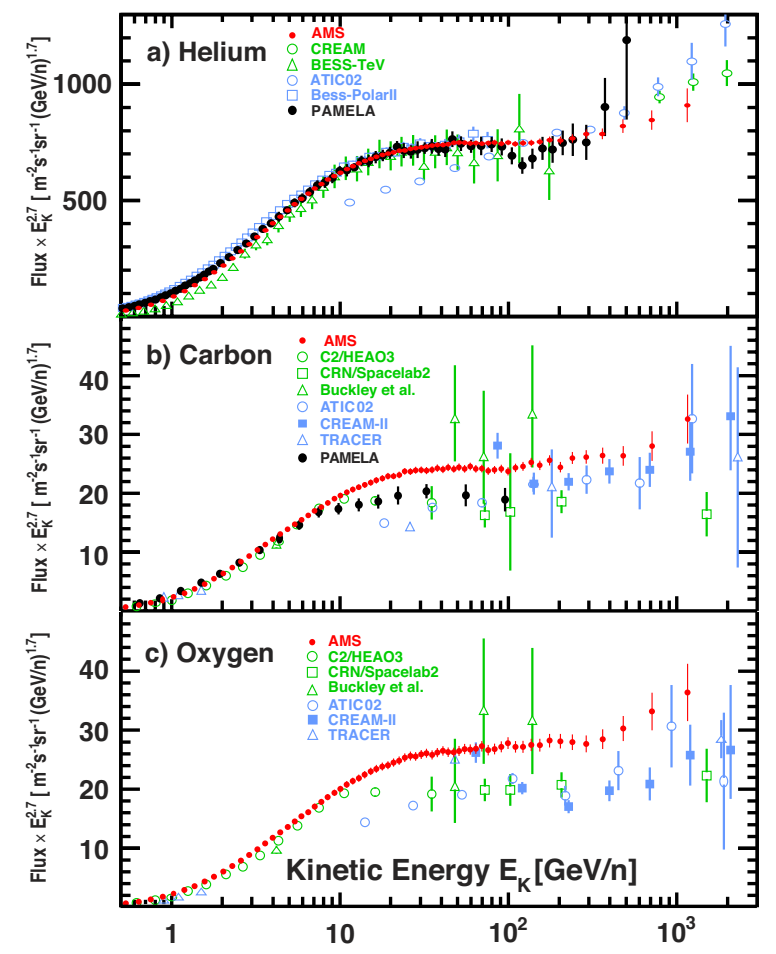

Figure 11. The AMS (a) helium, (b) carbon, and (c) oxygen fluxes as functions of kinetic energy per nucleon $E_{K}$ multiplied by $E_{K}^{2.7}$ together with earlier measurements [2-10].

due to the isotopic composition uncertainties were added in quadrature to the total errors. Figures 10, 11, 12, and 13 show the AMS light nuclei fluxes as a function of the kinetic energy per nucleon $E_{K}$ together with results of previous experiments [2-17]. As seen, AMS measurements measures with high precision all the light nuclei species.

In conclusion, a precise knowledge of the cosmic light nuclei fluxes with atomic number $1 \leq Z \leq 8$ is important in understanding the origin, acceleration, and propagation of cosmic rays. Precise measurement of AMS of the cosmic rays light nuclei fluxes in the rigidity range between $2 \mathrm{GV}$ to $3 \mathrm{TV}$ have been presented. Based on the ob-

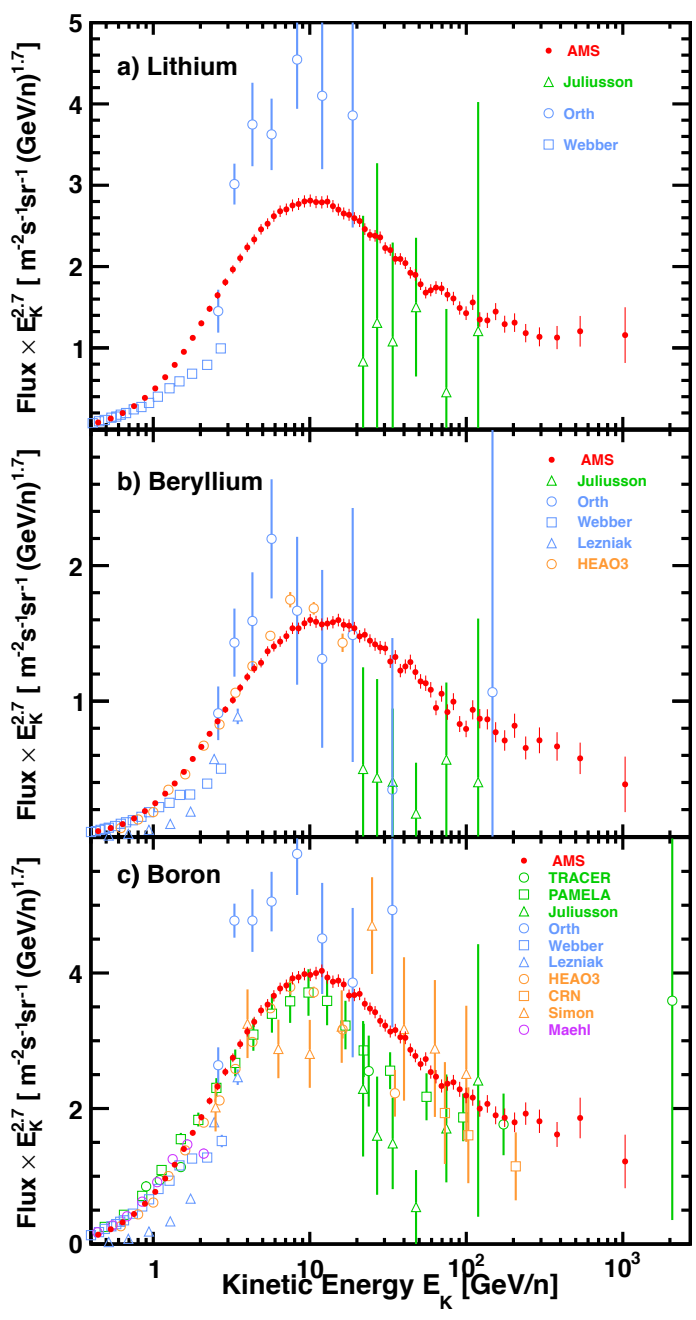

Figure 12. The AMS (a) lithium, (b) beryllium, and (c) boron fluxes as functions of kinetic energy per nucleon $E_{K}$ multiplied by $E_{K}^{2.7}$ together with earlier measurements $[3,6,7,10-16]$.

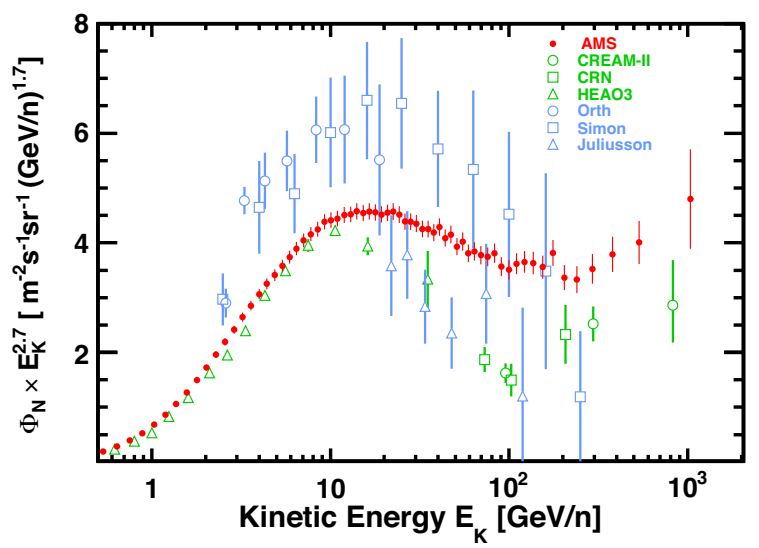

Figure 13. The AMS nitrogen flux $\Phi_{\mathrm{N}}$ as a function of kinetic energy per nucleon $E_{K}$ multiplied by $E_{K}^{2.7}$ [23] together with earlier measurements $[6,7,9,11,12,16]$.

served spectral behaviour, the light nuclei can be separated into three distinct families: primaries (hydrogen, helium, carbon, and oxygen), secondaries (lithium, beryllium, and 
boron), and mixed (nitrogen). Spectral indices of all light nuclei fluxes progressively harden above $100 \mathrm{GV}$. Primary cosmic ray fluxes have an identical hardening above 60 GV. While helium, carbon and oxygen have identical spectral index magnitude, the hydrogen spectral index shows a different magnitude, i.e. the primary-to-primary $\mathrm{H} / \mathrm{He}$ ratio is well described by a single power law above $45 \mathrm{GV}$ with index $-0.077 \pm 0.007$. Secondary cosmic ray fluxes have identical rigidity dependence above $30 \mathrm{GV}$. Secondary cosmic rays all harden more than primary species, and together all secondary-to-primary ratios show a hardening difference of $0.13 \pm 0.03$. Remarkably, the nitrogen flux is well described over the entire rigidity range by the sum of the primary flux equal to $(9 \pm 0.2) \%$ of the oxygen flux and the secondary flux equal to $(62 \pm 2) \%$ of the boron flux.

\section{References}

[1] For cosmic rays physics reviews see: I. A. Grenier et al., Annu. Rev. Astron. Astrophys. 53, 199 (2015); P. Blasi, Astron. Astrophys. Rev. 21, 70 (2013); A. W. Strong et al., Annu. Rev. Nucl. Part. Sci. 57, 285 (2007).

[2] For the BESS experiment see: K. Abe et al., Astrophys. J. 822, 65 (2016); Y. Shikaze et al., Astropart. Phys. 28, 154 (2007); S. Haino et al., Phys. Lett. B 594, 35 (2004); T. Sanuki et al., Astrophys. J. 545, 1135 (2000).

[3] For the PaMeLa experiment see: O. Adriani et al., Astrophys. J. 791, 93 (2014); O. Adriani, et al., Astrophys. J. 765, 91 (2013); O. Adriani, et al., Science 332, 69 (2011).

[4] For the CREAM-I experiment see: Y. S. Yoon et al., Astrophys. J. 728, 122 (2011); H. S. Ahn et al., Astrop. Phys. 30, 133 (2008).

[5] For the ATIC-02 experiment see: A. D. Panov et al., Bull. Russian Acad. Sci. 73, 564 (2009); A. D. Panov et al., Proc. of the 30th Int. Cosmic Ray Conf., Mérida, 2, 3 (2008); data were extracted using D. Maurin et al., Astron. Astrophys. 569, A32 (2014).

[6] J. J. Engelmann et al., Astron. Astrophys. 233, 96 (1990).

[7] S. P. Swordy, et al., Astrophys. J. 349, 625 (1990).

[8] J. Buckley, et al., Astrophys. J. 429, 736 (1994).

[9] For the CREAM-II experiment see: H. S. Ahn et al., Astrophys. J. 715, 1400 (2010); H. S. Ahn et al., Astrophys. J. 714, 89 (2010); H. S. Ahn et al., Astrophys. J. 707, 593 (2009).

[10] For the TRACER experiment see: A. Obermeier et al., Astrophys. J. 742, 14 (2011); M. Ave, et al., Astrophys. J. 678, 262 (2008) . F. Gahbauer et al., Astrophys. J. 607, 333 (2004).
[11] E. Juliusson, Astrophys. J. 191, 331 (1974).

[12] C. D. Orth et al., Astrophys. J. 226, 1147 (1978).

[13] W. R. Webber et al., Proc. of 16th Int. Cosmic Ray Conf., Tokyo, 12, 51 (1979).

[14] J. A. Lezniak et al., Astrophys. J. 223, 676 (1978).

[15] R. C. Maehl et al., Astrophys. Space Sci. 47, 163 (1977).

[16] M. Simon et al., Astrophys. J. 239, 712 (1980).

[17] M. Aguilar et al., Astrophys. J. 724, 329 (2010).

[18] M. Aguilar et al., Phys. Rev. Lett. 114, 171103 (2015).

[19] M. Aguilar et al., Phys. Rev. Lett. 115, 211101 (2015).

[20] M. Aguilar et al., Phys. Rev. Lett. 117, 231102 (2016).

[21] M. Aguilar et al., Phys. Rev. Lett. 119, 251101 (2017).

[22] M. Aguilar et al., Phys. Rev. Lett. 120, 021101 (2018).

[23] M. Aguilar et al., Phys. Rev. Lett. 121, 051103 (2018).

[24] P. L. Biermann et al., Astrophys. J. 725, 184 (2010); Y. Ohira and K. Ioka, Astrophys. J. Lett. 729, L13 (2011); Q. Yuan et al., Proc. of the 32nd Int. Cosmic Ray Conf., Beijing, 6, 222 (2011); S. Thoudam and J. R. Hörandel, Mon. Not. Royal Astron. Soc. 435, 2532 (2013).

[25] S. Thoudam and J. R. Hörandel, Astron. Astrophys. 567, A33 (2014); P. Blasi et al., Phys. Rev. Lett. 109, 061101 (2012); N. Tomassetti, Phys. Rev. D 92, 081301(R) (2015); A. E. Vladimirov et al., Astrophys. J. 752, 68 (2012).

[26] A. Kounine, Int. J. Mod. Phys. E 211230005 (2012); S. Rosier-Lees, in Proc. of Astrop. Phys. TEVPA/IDM, Amsterdam, 2014 (unpublished); S. Ting, Nucl. Phys. B, Proc. Suppl. 243-244, 12 (2013); S.-C. Lee, in Proc. of the 20th Int. Conf. on Supersymmetry and Unification of Fundamental Interactions (SUSY 2012), Beijing, 2012 (unpublished); M. Aguilar, in Proc. of the XL Int. Meeting on Fundamental Phys., Centro de Ciencias de Benasque Pedro Pascual, 2012 (unpublished); S. Schael, in Proc. of the 10th Symposium on Sources and Detection of Dark Matter and Dark Energy in the Universe, Los Angeles, 2012 (unpublished); B. Bertucci, Proc. Sci., EPS-HEP, (2011) 67; M. Incagli, AIP Conf. Proc. 1223, 43 (2010); R. Battiston, Nucl. Instrum. Methods Phys. Res., Sect. A 588, 227 (2008).

[27] K. Lübelsmeyer et al., Nucl. Instrum. Methods Phys. Res., Sect. A 654, 639 (2011).

[28] B. Alpat et al., Nucl. Instrum. Methods Phys. Res., Sect. A 613, 207 (2010); G. Ambrosi et al., Nucl. Instrum. Methods Phys. Res., Sect. A 869C, 29 (2017).

[29] V. Bindi et al., Nucl. Instrum. Methods Phys. Res., Sect. A 743, 22 (2014)

[30] J. Alcaraz et al., Phys. Lett. B 484, 10 (2000).

[31] C. C. Finlay et al., Geophys. J. Int. 183, 1216 (2010); E. Thébault et al., Earth Planets Space 67, 79 (2015). 